\title{
Enfoque terapéutico de la bacteriemia por Staphylococcus aureus
}

\author{
Diego Tibavizco ${ }^{1}$, José Yesid Rodríguez ${ }^{1}$, Edwin Silva ${ }^{2}$, Sonia Isabel Cuervo ${ }^{2,3}$, \\ Jorge Alberto Cortés ${ }^{1,4}$ \\ ${ }^{1}$ Departamento de Medicina, Facultad de Medicina, Universidad Nacional de Colombia, Bogotá, D. C., \\ Colombia \\ 2 Unidad de Infectología, Departamento de Medicina, Facultad de Medicina, Universidad Nacional de \\ Colombia, Bogotá, D. C., Colombia \\ ${ }^{3}$ Grupo de Infectología, Instituto Nacional de Cancerología, E.S.E., Bogotá, D. C., Colombia \\ ${ }^{4}$ Grupo para el Control de la Resistencia Bacteriana en Bogotá (GREBO), Bogotá, D. C., Colombia
}

Staphylococcus aureus es un patógeno importante que causa cerca de $11 \%$ a $33 \%$ de las bacteriemias hospitalarias y un porcentaje importante de las adquiridas en la comunidad, con una tasa de complicaciones cercana a 50\%. En la siguiente revisión se destaca la epidemiología de la bacteriemia por $S$. aureus, con especial referencia a la situación de este patógeno en Colombia, la frecuencia y los mecanismos de resistencia a los medicamentos más frecuentemente usados en este contexto, y se discuten los elementos semiológicos, clínicos y de laboratorio que influyen en el enfoque diagnóstico y terapéutico de los pacientes con bacteriemia por este microorganismo.

Palabras clave: Staphylococcus aureus/patogenicidad, bacteriemia/terapia, bacteriemia/ epidemiología, agentes antibacterianos/uso terapéutico.

\section{Therapeutic approach to Staphylococcus aureus bacteremia}

\begin{abstract}
Staphylococcus aureus is an important human pathogen, responsible for $11-33 \%$ of the bacteremias acquired in the hospital setting and nearly $50 \%$ of those acquired in the community at large. The epidemiology of $S$. aureus bacteremia is discussed, with an special emphasis on the situation in Colombia and the resistance mechanisms against the major drug groups used for the treatment. The clinical keys and laboratory support for the appropriate clinical approaches are presented together with the therapeutic strategies for the treatment of patients with $S$. aureus bacteremia.
\end{abstract}

Key words: Staphylococcus aureus/pathogenicity, bacteremia/therapy, bacteremia/ epidemiology, anti-bacterial agents/therapeutic use

Staphylococcus aureus es un patógeno importante tanto a nivel hospitalario como de la comunidad (1). En los Estados Unidos, S. aureus junto con Escherichia coli son los microorganismos más comúnmente aislados como

\footnotetext{
Correspondencia:

Jorge Alberto Cortés, Oficina 318, Departamento de Medicina, Edificio Facultad de Medicina, Universidad Nacional de Colombia, Bogotá, D. C.

Teléfono: (1) 316 5000, extensión 15011 y 15012; fax: (1) 316 5000, extensión 15011

jorgecortes@yahoo.com
}

Recibido: 09/10/07; aceptado: 30/04/07 patógenos hospitalarios; es la causa más común de neumonía hospitalaria e infección del sitio operatorio y la segunda causa de bacteriemia después de Staphylococci negativos para coagulasa (2). En la comunidad, persiste como una causa muy importante de infección de piel y de tejidos blandos, de infecciones respiratorias y de endocarditis infecciosa (especialmente entre usuarios de drogas inyectadas) (3-8). En Bogotá, durante el año 2002, $S$. aureus fue el principal microorganismo aislado en infecciones hospitalarias en $25,7 \%$ de los casos (9). Entre las infecciones hospitalarias asociadas a dispositivos 
invasivos en Bogotá, en unidades de cuidado intensivo entre el 2002 y el 2005, S. aureus ocupó el primer lugar de frecuencia con un porcentaje similar y una frecuencia de resistencia a los betalactámicos de $64 \%(9,10)$. Es de resaltar que las bacteriemias por este microorganismo son una causa muy importante de morbimortalidad, no sólo en nuestro medio sino también a nivel global, y se ha reportado un aumento de su incidencia en las últimas décadas (1).

El objetivo de esta revisión es describir el abordaje diagnóstico y terapéutico de la bacteriemia por $S$. aureus, resaltando los puntos que consideramos fundamentales para el análisis y la selección del tratamiento más apropiado de acuerdo con la epidemiología de las bacteriemias en nuestro medio, los mecanismos de resistencia antibiótica, el lugar geográfico de la adquisición de la bacteriemia, sus complicaciones y los medicamentos de que disponemos en la actualidad.

\section{Epidemiología de la bacteriemia por S. aureus}

A partir de la década de los 80 se ha incrementado el número de bacteriemias producida por $S$. aureus, tanto adquiridas en la comunidad como hospitalarias, fenómeno explicado por el aumento de la supervivencia de la población general, el aumento de enfermedades subyacentes, la aparición de ciertos hábitos sociales (uso de drogas endovenosas) e, incluso, la aplicación de los avances tecnológicos y terapéuticos en la medicina, especialmente el uso de dispositivos intravasculares $(4,11)$. En los Estados Unidos, entre 1980 y 1990, se incrementaron los casos de bacteriemia por S. aureus en $283 \%$ en hospitales no universitarios y, en $176 \%$, en hospitales universitarios $(7,12,13)$.

Según el lugar de adquisición de la bacteriemia por $S$. aureus se encuentra que $17 \%$ a $49 \%$ se originan en la comunidad, $51 \%$ a $83 \%$ son hospitalarias y $30 \%$ a $38 \%$ están asociadas con trabajadores de la salud (ver más adelante) (13-17).

S. aureus resistente a meticilina (SARM) es más frecuente en la bacteriemia hospitalaria, con tasas que varían de acuerdo con la localización geográfica $(5,15,18-20)$. Según datos del 2003 del Sistema Nacional de Vigilancia de las Infecciones
Hospitalarias (National Nosocomial Infections Surveillance System, NNIS) en los Estados Unidos, $59,5 \%$ de los aislamientos de $S$. aureus en pacientes con infecciones hospitalarias en unidades de cuidados intensivos, corresponden a SARM. Al comparar la tasa de resistencia entre enero y diciembre de 2003 con la observada en los cinco años previos, se evidenció un incremento de $11 \%$ (21). Según datos suministrados por el Grupo para el Control de la Resistencia Bacteriana en Bogotá al tener en cuenta todos los aislamientos, la resistencia a meticilina de $S$. aureus para 2001,2002 , y 2003 fue de $41 \%, 49 \%$ y $48 \%$, respectivamente $(9,22,23)$.

De las bacteriemias por $S$. aureus detectadas en el momento de la hospitalización, $12 \%$ a $39 \%$ son causadas por SARM según la serie; cuando en estos pacientes se evalúa la presencia de factores de riesgo para bacteriemia relacionada con trabajadores de la salud, hasta $97 \%$ cumplen con al menos un criterio, es decir, sólo cerca de 3\% de las bacteriemias por SARM detectadas al ingreso al hospital podrían ser consideradas como verdaderamente adquiridas en la comunidad (5,6,24-27). En Bogotá, 2,8\% de los SARM aislados de pacientes ambulatorios presentan fenotipo sugestivo adquirido en comunidad (sensible a eritromicina y clindamicina) (Cortés JA, Grupo para el Control de la Resistencia Bacteriana en Bogotá, comunicación personal).

Los estudios de análisis de costos informan que cada caso de bacteriemia hospitalaria produce estancias hospitalarias que varían entre 7 y 30 días, con un incremento en los costos de atención por sobreviviente entre US $\$ 5.000$ y US $\$ 40.000$, los cuales son más elevados si el microorganismo es resistente a la meticilina (28-31).

Mecanismos de resistencia antibiótica del $S$. aureus

Existen múltiples opciones terapéuticas por considerar en el tratamiento de la bacteriemia por $S$. aureus. Sin embargo, con el problema creciente de la resistencia a los antibióticos es fundamental recordar los principales mecanismos reconocidos en $S$. aureus, con el fin de seleccionar más racionalmente el uso de antibióticos en esta entidad clínica. 
Los mecanismos de resistencia antibiótica del $S$. aureus que se describen a continuación se relacionan con el uso cronológico de los medicamentos que se han utilizado.

1. Producción de penicilinasas. Las bacteriemias por $S$. aureus presentaban tasas de mortalidad que se acercaban al $80 \%$, y hasta el $70 \%$ desarrollaban metástasis infecciosas en la era preantibiótica $(1,16,32)$. La introducción de las benzilpenicilinas al ámbito clínico en 1941, produjo una mejoría notable en el pronóstico de los pacientes, pero en 1942 se identificaron las primeras cepas de $S$. aureus resistentes a la penicilina. Para $1960,80 \%$ de las cepas hospitalarias y adquiridas en la comunidad eran resistentes a la penicilina. Actualmente, menos de $10 \%$ de las cepas adquiridas en la comunidad y muy pocas de las cepas hospitalarias son sensibles a la penicilina G $(33,34)$.

La resistencia a la penicilina se explica por la inactivación del fármaco (hidrolización del anillo beta-lactámico) a través de la producción de una enzima conocida como beta-lactamasa, la cual es codificada por el gen blaZ, que hace parte de un elemento transportable localizado en un plásmido que, usualmente, confiere resistencia a otros antibióticos (35). La expresión del gen blaZ está condicionada por dos genes reguladores blaR1 (antirrepresor) y blal(represor). Actualmente, basados en la hipótesis enunciada por Zhang et al., se cree que la exposición de la bacteria al fármaco beta-lactámico hace que blaR1, actuando como sensor-transductor trans-membrana, se escinda a sí mismo, y los residuos actúen como proteasas que, finalmente, dividen el blalpermitiendo que el gen blaZ sintetice la enzima (36).

2. Gen mecA (SARM). La meticilina es una penicilina semisintética resistente a la betalactamasa, la cual fue introducida a la práctica clínica en 1959 como solución a la infección producida por $S$. aureus resistente a la penicilina; sin embargo, sólo dos años después de su introducción se reportaron los primeros casos de cepas resistentes a este antibiótico (34). El porcentaje de resistencia a la meticilina se ha incrementado velozmente y se explica en parte por la selección generada por el uso inapropiado de antibióticos beta-lactámicos. Actualmente, hay gran preocupación por la presencia cada vez mayor de SARM adquirido en la comunidad en pacientes que no han tenido contacto previo con hospitales, ni con un factor de riesgo conocido (37).

La resistencia a la meticilina es conferida por el gen $m e c A$, el cual es responsable de la síntesis de la proteína ligadora de penicilina 2a (PBP2a o PBP2'). Las PBP son enzimas ligadas a la membrana, las cuales catalizan la reacción de transpeptidación necesaria para unir las cadenas de peptidoglicanos. Las PBP2a presentan baja afinidad a los antibióticos beta-lactámicos, por lo tanto, permiten la supervivencia de la bacteria en ambientes con alta concentración de estos agentes; cuando está presente el gen mecA (SARM), se confiere resistencia a todos los antibióticos beta-lactámicos, inclusive las cefalosporinas.

El mecA hace parte de una isla genómica conocida como casete cromosómico estafilocóccico (SCCmec, por sus siglas en inglés, Staphylococcal Cassette Chromosome mec) el cual se integra al cromosoma de $S$. aureus. Actualmente, se han caracterizado cinco tipos diferentes de SCCmec, los cuales difieren en tamaño y composición genética. Basados en el tipo de SCCmec y en análisis filogenéticos, las cepas de SARM se pueden clasificar como hospitalarias si presentan SCCmec tipo I, II o III y, adquiridas en la comunidad, cuando presentan SCCmec tipo IV o V.

EI SCCmec puede transportar en forma variable genes de resistencia a otros antibióticos como aminoglucósidos, clindamicina, eritromicina, rifampicina, tetraciclina y trimetoprimsulfametoxazol (16,32-34); los SARM adquiridos en el hospital son los que presentan más comúnmente resistencia a estos antibióticos. La expresión fenotípica de estos perfiles de sensibilidad antibiótica se ha utilizado como una herramienta más para clasificar el origen de las cepas de SARM.

De igual forma, SARM puede clasificarse en clones según el patrón de electroforesis de campo de pulso en gel (que guarda relación con el SSCmec y, por ende, con patrón de susceptibilidad antibiótica). Estos estudios permiten identificar 
el patrón de resistencia característico en un área o región.

En un estudio llevado a cabo en Bogotá entre 1996 y 1998, se evidenció que el clon predominante era el patrón $\mathrm{D}$ (pediátrico) de electroforesis de campo pulsado en gel, mientras que en el resto de Latinoamérica el patrón predominante era el $\mathrm{B}$ (brasilero). En otro estudio realizado en siete ciudades de Colombia entre 2001 y 2002 se evidenció que hubo un cambio en el clon predominante, y el patrón $\mathrm{F}$ (chileno) se tornó el más importante $(68,5 \%)$. Este patrón presenta una resistencia muy alta a gentamicina $(98,5 \%)$, ciprofloxacina (92\%), macrólidos y lincosamidas, con una resistencia intermedia a rifampicina (20\%) y susceptibilidad a trimetoprim-sulfametoxazol (mayor de 90\%). Lo anterior sugiere que la rifampicina y el trimetoprim-sulfa son alternativas útiles para el manejo de infecciones por SARM, mientras que las fluoroquinolonas y los aminoglucósidos tienden a ser inefectivos en nuestra población (38-40), a diferencia de lo que ocurre en otros países, como los Estados Unidos (33).

3. Mutaciones espontáneas en los cromosomas que codifican la producción de topoisomerasa IV o ADN girasa. Las quinolonas se han usado para tratar infecciones por bacterias Gram positivas. La resistencia de $S$. aureus contra las quinolonas apareció rápidamente, especialmente entre las cepas SARM. En muchas instituciones se evidenció el incremento de la resistencia de $S$. aureus a las quinolonas de $7 \%$, en 1988 , a $83 \%$ en 1990. La resistencia a las quinolonas se explica por mutaciones espontáneas en los genes que codifican la producción de topoisomerasa IV o ADN girasa, los que producen cambios de aminoácidos de regiones críticas de dichas enzimas y, así, alteran su afinidad por el fármaco. GrIA de la topoisomerasa (en Staphylococci, parC en otras bacterias) y gyrA de la girasa son los sitios más comunes de mutación que producen resistencia. Entre los Gram positivos las mutaciones suelen suceder con mayor frecuencia en el gen de la topoisomerasa (41). Otro mecanismo de resistencia es la introducción de una bomba de salida de resistencia multiantibióticos. Además, el uso de fluoroquinolonas se asocia con un aumento del riesgo de adquisición hospitalaria de infección por S. aureus (34). La moxifloxacina y la gatifloxacina son más potentes contra $S$. aureus y presentan menos probabilidad de seleccionar cepas resistentes (1).

4. Alteración de la pared celular Vs. transferencia de plásmido que contiene los genes vanA. La terapia de elección para el tratamiento de infecciones graves por SARM es la vancomicina, por lo que su uso en la práctica clínica es cada día más frecuente. Su mecanismo de acción es la inhibición de la síntesis de la pared celular al unirse con las terminaciones D-ala-D-ala de las unidades precursoras de la pared; así, inhibe las reacciones de polimerización de péptido-glicanos y la transpeptidación, con lo cual se previene el entrecruzamiento de peptidoglicanos de la pared celular en la segunda fase de su síntesis.

En 1997 se informó el primer caso de S. aureus con resistencia intermedia a la vancomicina y en los últimos años ya se han registrado casos de cepas resistentes a la vancomicina $(42,43)$. En $S$. aureus con resistencia intermedia a la vancomicina el mecanismo de resistencia consiste en el aumento del grosor de la pared celular a través del aumento en el número de capas de peptidoglicano que la componen, lo cual hace que se atrape más vancomicina en las capas superficiales y evita que el fármaco llegue a la membrana citoplasmática. En las cepas resistentes a la vancomicina, el mecanismo de resistencia resultó de la transferencia del operón vanA desde Enterococcus faecalis resistente a vancomicina, el cual produce alteración del péptido terminal de D-ala-D-ala a D-ala-D-Lac, el cual sólo se produce cuando la bacteria se expone a concentraciones bajas de vancomicina (44-47).

5. Resistencia frente a tetraciclinas. Las tetraciclinas actúan sobre la unidad 305 ribosómica, al inhibir la elongación en la síntesis de proteínas. El principal mecanismo de resistencia es a través de la salida activa del fármaco a través de una proteína codificada por un plásmido (tet $K$ y tet $L$ ); en menor medida, a través de la modificación del ribosoma (tet $M$ y tet $O$ ). Este mecanismo no afecta a la tigeciclina $(1,32,33)$.

6. Resistencia frente a trimetoprim-sulfametoxazol. Trimetoprim y sulfametoxazol son dos 
antimetabolitos que inhiben la síntesis de tetrahidrofolato, el cual es esencial para la síntesis de timidina, purina, ADN y algunos aminoácidos. EI sulfametoxazol inhibe competitivamente la enzima dihidropteroato-sintetasa y la resistencia se puede producir por cambio estructural en la enzima, o producción excesiva de ácido paraaminobenzoico $(d p s A)$. Por otra parte, el trimetoprim inhibe competitivamente la enzima dihidrofolatoreductasa y el principal mecanismo de resistencia es una dihidrofolato reductasa alterada, originaria en un transposón (dfrA) $(32,33)$.

7. Resistencia frente a linezolid. El linezolid es un antibiótico relativamente nuevo de la clase de las oxazolidinonas, que actúa a través de la inhibición del complejo de iniciación en la subunidad 30 S ribosómica, evitando la unión del ARNm e impidiendo el inicio de la traslación. El mecanismo de resistencia antibiótico descrito consiste en mutación del gen que codifica para rARN de $23 S(1,32,33)$.

\section{Evaluación del paciente con bacteriemia por S. aureus}

Los factores relevantes que deben considerarse al evaluar un paciente con bacteriemia por $S$. aureus son los siguientes.

\section{Lugar de adquisición de la bacteriemia}

El lugar de adquisición de la infección tradicionalmente se ha dividido entre adquirido en la comunidad y hospitalario, distinción importante pues sobre ella se define la probabilidad de resistencia de $S$. aureus y, por lo tanto, la conducta terapéutica y el pronóstico.

La bacteriemia adquirida en la comunidad corresponde a la que se identifica en las primeras 48 horas de hospitalización, en el paciente que no haya estado hospitalizado durante los siete días precedentes al motivo de consulta. La bacteriemia hospitalaria corresponde a la que es identificada después de las 48 horas de hospitalización y durante los siete días posteriores al egreso del hospital.

Recientemente, se ha descrito un tercer grupo denominado bacteriemias asociadas a trabajadores de la salud, que se define como las bacteriemias que, a pesar de ser adquiridas en la comunidad, están asociadas con terapia endovenosa en casa, cuidado de heridas o cuidado de enfermería especializada, hemodiálisis o quimioterapia en los 30 días previos, hospitalización por dos o más días en hospital de cuidado agudo en los últimos 90 días o vivir en un ancianato o clínica de reposo; su importancia radica en que tiene un comportamiento microbiológico de resistencia similar al de las bacteriemias hospitalarias $(5,34,48)$.

\section{Diagnóstico topográfico de la bacteriemia}

La bacteriemia por $S$. aureus puede clasificarse como primaria o secundaria, según se identifique o no el órgano o sistema infectado (16).

El órgano o sistema origen de la bacteriemia está muy relacionado con el lugar en el que se adquiere la infección (48). En el estudio de Jensen et al. (6), se determinó que los catéteres intravenosos $(45 \%)$, las heridas quirúrgicas (16\%) y el aparato respiratorio $(13 \%)$ fueron las principales puertas de entrada para la bacteriemia hospitalaria. En el caso de la bacteriemia adquirida en la comunidad los focos de origen fueron principalmente las lesiones en piel (40\%), seguidas por el aparato respiratorio (18\%). Este mismo estudio mostró que $4 \%$ de las bacteriemias hospitalarias y $20 \%$ de las adquiridas en la comunidad fueron primarias.

\section{Complicaciones de la bacteriemia por $S$. aureus}

La frecuencia de las complicaciones varía entre $11 \%$ y $53 \%$, las cuales se dividen en recurrencia (6\% a 12\%), enfermedad metastásica (hasta $31 \%$ ) y mortalidad $(6,49)$. La tasa de mortalidad atribuible a bacteriemias por $S$. aureus no ha cambiado en las últimas décadas, persiste entre $11 \%$ y $43 \%(6,50,51)$. Las principales metástasis infecciosas son: endocarditis $73 \%$, artritis séptica $26 \%$ y osteomielitis $26 \%$, pero también, se ha descrito compromiso del sistema nervioso central (meningitis, abscesos intracerebrales o epidural), neumonías, abscesos de tejidos blandos e infección de vías urinarias (figura 1) $(6,16,33,52,53)$.

\section{Factores de riesgo y pronósticos}

En los diversos estudios realizados se han identificado múltiples factores relacionados con 


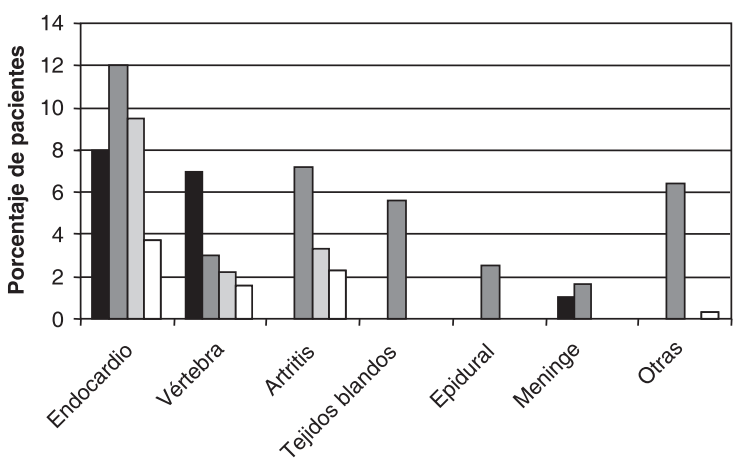

Jensen $2002 \square$ Fowler $2003 \quad \square$ Fowler $2005 \square$ Melzer 2003 $\begin{array}{llll}(278 \mathrm{pts}) & \text { (724 pts) } & \text { (324 pts) } & \text { (815 pts) }\end{array}$

Figura 1. Porcentaje de pacientes con metástasis infecciosas secundarias a bacteriemia por $S$. aureus según diferentes estudios.

Endocardio: endocarditis infecciosa; vértebra: osteomielitis vertebral; artritis: artritis séptica; tejidos blandos: abscesos de tejidos blando; epidural: absceso epidural; meninge: meningitis; otras: otras. Construido a partir de los datos de las referencias $6,16,52$ y 53 .

aumento de la morbimortalidad, asociada con la bacteriemia por $S$. aureus $(12,15,17,18,30,54,55)$. Se han identificado factores del huésped que se asocian con un desenlace adverso, como edad mayor de 60 años e inicio de la bacteriemia en la comunidad (56-60). En este sentido también se han encontrado elementos de la anamnesis o del examen físico, como la presencia de cuerpos extraños o prótesis (52), la incapacidad de identificar el foco de la bacteriemia $(12,51,59,61)$, la morbilidad asociada grave y previa $(52,56,59,62)$, la presencia de lesiones vasculares cutáneas (16), las metástasis infecciosas y la presencia de choque séptico al ingreso (6).

Entre los factores relacionados con el tratamiento, se han identificado la resistencia a la meticilina $(55,63,64)$, el retraso en el diagnóstico $(52)$, la persistencia de cuerpos extraños $(65,66)$ o focos no removibles $(52,56)$, la persistencia de la fiebre después de 72 horas $(16,56)$ y el retraso en la administración del antibiótico apropiado por más de 44 horas (63), como factores que predicen una mayor probabilidad de fracaso terapéutico, siembras metastásicas y recurrencia. La mayoría de estos estudios corresponden a estudios de cohorte, con tiempos variables de seguimiento. De acuerdo con los análisis multivariados, una tasa de complicaciones cercana al $35 \%$ se asocia con alguna de las siguientes características: 1) adquirida en la comunidad, 2) hallazgos en piel sugestivos de infección sistémica aguda, 3) hemocultivo de control positivo, usualmente a las 72 horas y 4) persistencia de la fiebre a las 72 horas de iniciado el tratamiento (16).

\section{Resistencia a la meticilina}

Algunos estudios han demostrado incremento en la mortalidad en pacientes con bacteriemia por SARM. Sin embargo, no se han evidenciado diferencias significativas en la tasa de diseminación al comparar SARM con S. aureus sensible a la meticilina, lo cual sugiere que, aunque la virulencia sea similar, la resistencia a los betalactámicos puede ser un factor determinante en la mortalidad como consecuencia del retraso en la elección del medicamento apropiado o por las diferencias en la actividad de los antibióticos frente al microorganismo $(53,64-66)$.

\subsection{SARM adquirido en la comunidad}

EI SARM que es verdaderamente adquirido en la comunidad presenta características epidemiológicas, genotípicas y fenotípicas que permiten diferenciarlo del asociado con trabajadores de la salud y del hospitalario, como son: ausencia de factores de riesgo asociados al hospital, resistencia antibiótica limitada a los betalactámicos, presencia del SCCmec tipo 4 y de genes que codifican para toxinas como la leucocidina de Pantone-Valentine y otras enterotoxinas $(67,68)$.

En pacientes que presentan bacteriemia por $S$. aureus desde el ingreso al hospital, se debe evaluar el riesgo de resistencia a la meticilina diferenciando aquéllos con criterios de bacteriemia asociada a trabajadores de la salud del subgrupo de bacteriemias verdaderamente adquiridas en la comunidad, ya que, como se comentó con anterioridad, hasta $97 \%$ de las bacteriemias por SARM detectadas al ingreso a un hospital cumplen criterios para considerarse asociadas a trabajadores de la salud.

En cuanto a las bacteriemias con verdadero origen en la comunidad, no están claramente establecidos los factores de riesgo para presentar 
SARM. Sin embargo, se ha visto que SARM adquirido en comunidad infecta más a pacientes jóvenes, habitantes de la calle, usuarios de drogas IV o con antecedentes de consumo previo de antibióticos $(37,67)$.

\section{Enfoque diagnóstico y terapéutico del paciente con bacteriemia por S. aureus}

\section{Construcción del diagnóstico}

El tratamiento de la bacteriemia por $S$. aureus tiene como objetivo erradicar la bacteriemia y prevenir la recaída o las metástasis infecciosas. Es importante en el momento del diagnóstico, identificar si la bacteriemia es primaria o secundaria, el lugar de adquisición de la bacteriemia, evaluar la presencia de cuerpos extraños, de colecciones (abscesos) o de tejido desvitalizado, y descartar la presencia de metástasis infecciosas $(69,70)$.

La evaluación y el seguimiento clínico continúan siendo de vital importancia, por lo cual se debe realizar un examen físico acucioso, ya que es la guía para la solicitud de otros métodos diagnósticos. Por ejemplo, en el caso de endocarditis, es importante buscar activamente los criterios clínicos (tanto vasculares como inmunológicos o la aparición de un nuevo soplo de insuficiencia) que permitan establecer un diagnóstico posible o definitivo de endocarditis infecciosa aplicando los criterios de Duke $(7,15,71,72)$ (cuadros 1 y 2 ). De igual forma, se recomienda la realización de un hemocultivo de control a las 72 horas y, si es positivo, que se busquen los posibles focos infecciosos metastáticos.

Ante la sospecha de endocarditis, ¿cuándo se debe solicitar el ecocardiograma?

La endocarditis infecciosa es una de las complicaciones más frecuentes asociadas con la bacteriemia por $S$. aureus. Los reportes de los estudios evidencian que hasta $32 \%$ de las endocarditis como hallazgo de necropsia no se sospechan clínicamente y, por otro lado, que 18\% de los pacientes con bacteriemia por $S$. aureus sometidos a ecocardiograma rutinario (sin estigmas de endocarditis infecciosa) tienen evidencia ecocardiográfica de ésta. Es por esto que, en estudios de analisis de decisiones, al excluir la presencia de endocarditis infecciosa con el uso de esta herramienta diagnóstica, se reduciría en $60 \%$ la duración de los tratamientos antibióticos y se lograría una disminución en los costos $(7,15,69,70)$.

Vale la pena mencionar que estos estudios de análisis de decisiones no son aplicables a nuestro medio, donde los costos son considerablemente más bajos. De otra parte, el ecocardiograma transesofágico es una herramienta diagnóstica con una sensibilidad y una especificidad adecuadas (sensibilidad cercana a 90\%) para identificar endocarditis infecciosa en la población general y en grupos especiales como son los pacientes ancianos, en presencia de válvulas protésicas, en endocarditis de cavidades derechas y en presencia de marcapasos o dispositivos intravasculares.

Para resumir, podemos afirmar que el ecocardiograma está indicado en: 1) endocarditis infecciosa probable, 2) hemocultivo de control positivo, 3) persistencia de fiebre luego de 72 horas de tratamiento antibiótico adecuado, 4) antecedentes de enfermedad valvular, y 5) presencia de prótesis valvular. Sin embargo, algunos autores recomiendan realizar ecocardiograma transtorácico a todos los pacientes con bacteriemia por $S$. aureusy, si presentan una probabilidad alta antes de prueba o múltiples factores de riesgo para complicaciones hematógenas, se recomienda de entrada realizar ecocardiograma transesofágico (7). No existen estudios clínicos que hayan demostrado el beneficio de esta estrategia.

\section{Propuesta de antibioticoterapia}

La aparición de cepas de $S$. aureus resistentes a penicilina, meticilina y otros antibióticos de amplio espectro hace cada día más difícil la escogencia de un antibiótico en forma empírica. Si la cepa aislada es sensible a la penicilina $(<10 \%$ de las cepas), el tratamiento de elección es penicilina G. Si la cepa es resistente a la penicilina pero sensible a meticilina, la elección es oxacilina. Si el paciente es alérgico a penicilinas y la cepa es $S$. aureus sensible a la meticilina, una opción válida es el uso de cefalosporinas, a menos que la alergia sea de tipo anafiláctico, en cuyo caso está indicado el uso de vancomicina. 
Cuadro 1. Criterios de Duke modificados para el diagnóstico de endocarditis infecciosa.

\section{Criterios mayores}

\section{Microbiológicos}

Microorganismos típicos consistentes con endocarditis infecciosa aislados de dos hemocultivos separados: Streptococcus viridans, Streptococcus bovis, grupo HACEK, Staphylococcus aureus; o bacteriemia por enterococo adquirida en la comunidad, en ausencia de un foco primario

\section{$\mathrm{O}$}

Microorganismos consistentes con endocarditis infecciosa aislados de cultivos persistentemente positivos, definidos así: a) al menos, dos hemocultivos positivos con $>12$ horas de diferencia entre la toma; b) todos de tres o la mayoría de $>4$ hemocultivos separados (al menos, con una hora de diferencia entre el primero y el último)

O

Único hemocultivo positivo para Coxiella burnetii o títulos de anticuerpos $\lg G>1: 800$

\section{Evidencia de compromiso endocárdico}

Regurgitación valvular nueva (no es suficiente el incremento o cambio en soplo preexistente)

Ecocardiograma positivo para endocarditis infecciosa: masa intracardiaca oscilante, ecogénica, sobre la válvula o estructuras de soporte localizada en sitio de daño endocárdico o sobre material implantable, absceso perianular o dehiscencia nueva de válvula protésica. (Se recomienda ecocardiograma transesofágico en pacientes que tienen válvula protésica, en quienes tienen "posible endocarditis infecciosa" por criterios clínicos o quienes presentan endocarditis infecciosa complicada)

\section{Criterios menores}

Predisposición a endocarditis infecciosa que incluye ciertas condiciones cardiacas o usuarios de drogas IV

Fiebre. Temperatura $>38^{\circ} \mathrm{C}$

Fenómenos vasculares. Émbolo arterial mayor, infartos pulmonares sépticos, aneurismas micóticos, hemorragia intracraneal, hemorragia conjuntival o lesiones de Janeway (las petequias o hemorragias en astilla se excluyen).

Fenómenos inmunológicos. Presencia de factor reumatoideo, glomerulonefritis, nódulos de Osler o mancha de Roth

Hallazgos microbiológicos. Hemocultivos positivos que no cumplen los criterios mayores o evidencia serológica de infección activa con organismo consistente con endocarditis infecciosa

Cuadro 2. Interpretación de los criterios de Duke para el diagnóstico de endocarditis infecciosa.

\section{El definitivo Criterios patológicos}

Microorganismos demostrados por cultivo o histología de una vegetación que ha producido émbolos o un absceso intracardiaco

Lesión patológica: vegetación o absceso intracardiaco confirmado por examen histológico que muestra endocarditis activa

\section{Criterios clínicos}

2 criterios mayores

1 criterio mayor y 3 criterios menores

5 criterios menores

El posible $\quad 1$ criterio mayor y 1 criterio menor

3 criterios menores

El descartado Diagnóstico alternativo que explique los hallazgos clínicos

Resolución de la clínica con $<4$ días de tratamiento antibiótico

Ausencia de evidencia patológica de endocarditis por cirugía o autopsia, con <de 4 días de tratamiento antibiótico

No cumple los criterios de posible endocarditis. 
Si el germen aislado es SARM, la elección es la vancomicina con dosis empírica de $30 \mathrm{mg} / \mathrm{kg}$ al día, dividida en 2-4 dosis diarias, buscando obtener niveles séricos aproximados de $30 \mathrm{mg} / \mathrm{ml}$ y ajustada a la función renal $(33,54,57)$. Debido a la aparición de cepas de $S$. aureus con resistencia intermedia a la vancomicina o SARV, se han introducido nuevos antibióticos, entre los que se encuentran linezolid (familia oxazolidinona), quinupristin/dalfopristin (familia estreptogramina, no disponible en Colombia), daptomicina (lipopéptido cíclico, no disponible en Colombia), entre otros $(1,13,15,33,-34,54,63,64,71,73)$; no hay que olvidar otros antibióticos como trimetoprim-sulfametoxazol, clindamicina, rifampicina, fluoroquinolonas, teniendo en mente que el gen MecA puede conferir resistencia variable a uno o varios de estos antibióticos $(15,18,54,63,64,71,74)$. Cabe recordar que la vancomicina no se considera tan efectiva como los beta-lactámicos, por lo que siempre que sea posible se debe preferir el uso de penicilinas antiestafilocóccicas antes que de vancomicina $(55,75)$ (cuadro 3).

Una vez que la incidencia de la resistencia de las cepas aisladas al antibiótico convencionalmente utilizado se sitúa entre $10 \%$ y $20 \%$, se sugiere iniciar de forma empírica nuevos agentes terapéuticos. Es por esto que la penicilina $G$ no se utiliza como primera elección a la hora de escoger tratamiento empírico para bacteriemia por $S$. aureus adquirido en la comunidad, en cuyo caso se prefiere el uso de oxacilina. Por la misma razón, una vez que se sospecha bacteriemia por $S$. aureus hospitalaria o asociada con el cuidado de la salud, la elección es vancomicina. Con el resultado del estudio microbiológico y del perfil de susceptibilidad se podrá decidir el cambio a una terapia antibiótica $(1,13,15,18,27,34,54,63$, 71,74).

Actualmente, se están realizando estudios para demostrar la efectividad del uso de linezolid como antibiótico de primera línea en el tratamiento de SARM. Hasta la fecha se ha demostrado por medio de un estudio clínico aleatorio y controlado, que el tratamiento con linezolid de pacientes con infecciones por SARM (incluso algunos pacientes
Cuadro 3. Antibióticos disponibles para el tratamiento de la bacteriemia por $S$. aureus en adultos.

\begin{tabular}{|c|c|c|}
\hline Antibiótico & Dosis & Frecuencia \\
\hline $\begin{array}{l}\text { S. aureus sensible a penicilina: } \\
\text { Penicilina G }\end{array}$ & 4'000.000 U & $4 \mathrm{~h}$ \\
\hline $\begin{array}{l}\text { S. aureus sensible a meticilina: } \\
\text { Oxacilina }\end{array}$ & $2 \mathrm{~g}$ & $4 \mathrm{~h}$ \\
\hline $\begin{array}{l}\text { S. aureus resistente a meticilina: } \\
\text { Vancomicina }\end{array}$ & $1 \mathrm{~g}$ & $12 \mathrm{~h}$ \\
\hline $\begin{array}{l}\text { S. aureus con resistencia interme } \\
\text { a vancomicina } \\
\text { Linezolid } \\
\text { Vancomicina (?) }\end{array}$ & $\begin{array}{l}\text { ledia } \\
\qquad \begin{array}{l}600 \mathrm{mg} \\
1 \mathrm{~g}\end{array}\end{array}$ & $\begin{array}{l}12 \mathrm{~h} \\
12 \mathrm{~h}\end{array}$ \\
\hline $\begin{array}{l}\text { S. aureus resistente a } \\
\text { vancomicina } \\
\text { Linezolid }\end{array}$ & $600 \mathrm{mg}$ & $12 \mathrm{~h}$ \\
\hline
\end{tabular}

con bacteriemia) es, por lo menos, tan efectivo como la vancomicina (75). Además, el linezolid es el antibiótico de elección cuando la cepa es $S$. aureus con resistencia intermedia a la vancomicina o SARV (cuya presencia no ha sido informada en Colombia) (56,62,73,75-80); algunos reportes preliminares sugieren que este antibiótico debe ser la elección en neumonía hospitalaria producida por este microorganismo $(69,74)$.

\section{Duración del tratamiento antibiótico}

La apropiada duración del tratamiento para bacteriemias causadas por $S$. aureus es un aspecto controvertido. Aunque la literatura mundial ha identificado múltiples factores de riesgo asociados con complicaciones y pronóstico en bacteriemias por este microorganismo, no existe consenso respecto a cuáles de estos factores son relevantes como definitorios de la duración del tratamiento. Los pocos estudios publicados presentan limitaciones metodológicas producidas especialmente por la heterogeneidad de los factores de riesgo evaluados y el bajo número de pacientes incluidos. La duración de la terapia antibiótica depende del diagnóstico clínico; es así como se recomiendan dos semanas de duración para bacteriemias no complicadas (paciente inmunocompetente, sin anomalía valvular, en quien el foco se retiró prontamente, con hemocultivo negativo a las 72 horas, sin evidencia de infección metastásica) y cuatro a 
seis semanas para las demás bacteriemias. En presencia de osteomielitis, algunos autores recomiendan tratamientos tan largos como ocho semanas o más $(7,15,69,81-84)$.

\section{Retiro del foco de origen de bacteriemia}

Tan importante como la elección del antibiótico apropiado para el éxito del tratamiento de la bacteriemia, son la remoción del foco de origen de la bacteriemia, el retiro de los cuerpos extraños y el drenaje de todas las colecciones supurativas o de tejidos desvitalizados (69). Varios estudios han demostrado que los casos en que se presenta bacteriemia asociada a un foco no erradicado, no identificable o con demora en retirarlo, se presenta mayor morbimortalidad $(6,12,17)$.

5. Prevención de la aparición de resistencia antibiótica y del desarrollo de la bacteriemia

El uso inadecuado de los antibióticos se ha señalado como uno de los factores principales en la aparición de cepas resistentes, por lo que una de las estrategias para prevenir su aparición es el uso prudente de los mismos $(34,79,80)$. La aparición y diseminación de la resistencia antibiótica es en gran parte responsabilidad de los trabajadores de la salud; por este motivo, es importante que todos ellos conozcan el problema y tomen conciencia para contener este grave problema $(56,85)$.

La solución para el control de la resistencia de $S$. aureus es multifactorial. Es importante hacer énfasis en la aplicación de estrategias de prevención de la diseminación de las cepas resistentes, entre las cuales se mencionan: 1) las medidas de aislamiento, que han demostrado ser efectivas en las diferentes instituciones, y 2) el lavado de manos, que ha demostrado ser eficaz en prevenir la transmisión de microorganismos de persona a persona.

\section{Vacuna}

Recientemente se han terminado ensayos clínicos que han demostrado que StaphVax, la vacuna que contiene los polisacáridos capsulares 5 y 8 , reduce la tasa de infecciones por $S$. aureus en $57 \%$ a 10 meses en un grupo de pacientes en hemodiálisis. Se cree que esta vacuna puede ser de gran importancia en otros grupos de pacientes de alto riesgo con catéteres permanentes, cirugía electiva, etc. También se han realizado estudios con otros compuestos como inmunoglobulina humana intravenosa para $S$. aureus (Altastaph), Tefibazumab (Aurexis; Inhibitex) y INH-A21 (Veronate; Inhibitex) buscando el mismo fin. Sin embargo, faltan estudios clínicos que sustenten estas hipótesis $(7,34,85)$.

\section{Conclusión}

$S$. aureus es un germen patógeno muy importante para el ser humano, no sólo por la amplia gama de enfermedades que produce, sino por el creciente aumento en su incidencia, el incremento en la resistencia antibiótica y su relación con un gran porcentaje de complicaciones y de mortalidad, aspectos que se reflejan en el aumento de los costos para el sistema de salud. Esta situación convoca a los trabajadores de la salud para conocer más específicamente este problema y los aspectos relacionados con la epidemiología, el diagnóstico y el tratamiento de una de las manifestaciones clínicas más frecuentes adquiridas en la comunidad o en el medio hospitalario: la bacteriemia por $S$. aureus.

El diagnóstico y enfoque terapéutico de las bacteriemias por este microorganismo incluyen un conocimiento apropiado del sitio de origen de la infección (localización geográfica y anatómica), de la evaluación de los factores de riesgo de complicaciones, de la selección apropiada de los antibióticos y de un seguimiento juicioso. Se requiere un mejor cumplimiento de las guías de lavado de manos y de aislamiento para disminuir la frecuencia de este problema clínico.

\section{Conflicto de intereses}

Los autores no tienen ningún conflicto de intereses que declarar.

\section{Financiación}

No hay fuente de financión directa para este manuscrito. Sin embargo, el mismo se realizó en el tiempo contratado por el Instituto Nacional de Cancerología y la Universidad Nacional de Colombia para Sonia Isabel Cuervo y para Jorge Alberto Cortés. 


\section{Referencias}

1. Kanafani ZA, Fowler VG. Staphylococcus aureus infections: new challenges from an old pathogen. Enf Infecc Microbiol Clin. 2006;24:182-93.

2. Emori TG, Gaynes RP. An overview of nosocomial infections, including the role of the microbiology laboratory. Clin Microbiol Rev. 1993;6:428-42.

3. Lowy FD. Staphylococcal infections. En: Kasper DL, Fauci AS, Longo DL, Braunwald E, Hauser SI, Jameson $\mathrm{JL}$, editors. Harrison's Principles of Internal Medicine. 16 ed. New York: Mc Graw Hill; 2005. p.814-22.

4. Elsayed S, Laupland K. Emerging gram-positive bacterial infections. Clin Lab Med. 2004;24:587-603.

5. Collignon P, Nimmo GR, Gottlieb T, Gosbell IB, Australian Group on Antimicrobial Resistance. Staphylococcus aureus bacteremia, Australia. Emerg Infect Dis. 2005;11:554-61.

6. Jensen AG, Wachmann $\mathbf{C H}$, Espersen $\mathbf{F}$, Scheibel J, Skinhoj P, Frimodt-Moller N. Treatment and outcome of Staphylococcus aureus bacteremia: a prospective study of 278 cases. Arch Intern Med. 2002;162:25-32.

7. Petti CA, Fowler VG Jr. Staphylococcus aureus bacteremia and endocarditis. Cardiol Clin. 2003;21:219-33.

8. Wisplinghoff $H$, Bischoff $T$, Tallent SM, Seifert $H$, Wenzel RP, Edmond MB. Nosocomial bloodstream infectios in US hospitals: analysis of 24179 cases from a prospective nationwide surveillance study. Clin Infect Dis. 2004;39:309-17.

9. Secretaria Distrital de Salud de Bogotá, D.C. Propuesta de Política de Prevención, Control, y Vigilancia Epidemiológica de Infecciones Intrahospitalarias para Bogotá DC. Mayo de 2004. [Consultado: noviembre 11 de 2006]. Disponible en http:// www.saludcapital.gov.co/secsalud/noticias/Politica.pdf

10. Moreno CA, Rosenthal VD, Olarte N, Gomez WV, Sussmann O, Agudelo J, et al. Device-associated infection rate and mortality in intensive care units of 9 colombian hospitals: findings of the international nosocomial infection control consortium. Infect Control Hosp Epidemiol. 2006;27:349-56.

11. Bearman GM, Wenzel RP. Bacteremias: a leading cause of death. Arch Med Res. 2005;36:646-59.

12. Hill PC, Birch M, Chambers S, Drinkovic D, EllisPegler RB, Everts R, et al. Prospective study of 424 cases of Staphylococcus aureus bacteremia: determination of factors affecting incidence and mortality. Intern Med J. 2001;31:97-103.

13. Linden PK. Clinical implications of nosocomial grampositive bacteremia and superimposed antimicrobial resistance. Am J Med. 1998;102:S24-33.

14. Banerjee S, Emori TG, Culver DH, Gaynes RP, Jarvis WR, Horan T, et al. Secular trends in nosocomial primary bloodstream infections in the United States. Am J Med. 1991;91:S86-9.

15. Chang FY, MacDonald BB, Peacock JE Jr, Musher DM, Triplett $\mathrm{P}$, Mylotte J M, et al. A Prospective multicenter study of Staphylococcus aureus bacteremia. Incidence of endocarditis, risk factors for mortality, and clinical impact of methicillin resistance. Medicine (Baltimore). 2003;82:322-32.

16. Fowler VG Jr, Olsen MK, Corey GR, Woods CW, Cabell CH, Reller LB, et al. Clinical identifiers of complicated Staphylococcus aureus bacteremia. Arch Intern Med. 2003;163:2066-72.

17. Kim SH, Park WB, Lee KD, Kang Cl, Kim HB, Oh MD, et al. Outcome of Staphylococcus aureus bacteremia in patients with eradicable foci versus noneradicable foci. Clin Infect Dis. 2003;37:794-9.

18. Soriano A, Martínez JA, Mensa J, Marco F, Almela M, Moreno-Martinez A, et al. Pathogenic significance of methicillin resistance for patients with Staphylococcus aureus bacteremia. Clin Infec Dis. 2000;30:368-73.

19. Fowler VG Jr, Sanders LL, Kong LK, McClelland RS, Gottlieb GS, Li J, et al. Infective endocarditis due to Staphylococcus aureus: 59 prospectively identified cases with follow-up. Clin Infect Dis. 1999;28:106-14.

20. Suryati BA, Watson M. Staphylococcus aureus bacteraemia in children: a 5-year retrospective review. J. Paediatr Child Health. 2002;38:290-4.

21. National Nosocomial Infections Surveillance System. National Nosocomial Infections Surveillance (NNIS) System Report, data summary from January 1992 through June 2004, issued October 2004. Am J Infect Control. 2004;32:470-85.

22. Leal A, Buitrago G, Castillo J, Patiño A, Álvarez C. Vigilancia de resistencia bacteriana. Resultados. Primer trimestre de 2005. Boletín Epidemiológico Distrital. 2005;10:1-8.

23. Leal AL, Eslava-Schmalbach J, Álvarez C, Buitrago G, Méndez M, Grupo para el Control de la Resistencia en Bogotá. Canales endémicos y marcadores de resistencia bacteriana en hospitales de tercer nivel en Bogotá, Colombia. Rev Salud Pública. 2006;8(Suppl.1):59-70.

24. Rezende NA, Blumberg HM, Metzger BS, Larsen NM, Ray SM, Mcgowan JE Jr. Risk factors for methicillin-resistance among patients with Staphylococcus aureus bacteremia at the time of hospital admission. Am J Med Sci. 2002;323:117-23.

25. Tacconelli E, Venkataraman L, De Girolami PC, D'Agata EM. Methicillin-resistant Staphylococcus aureus bacteraemia diagnosed at hospital admission: distinguishing between community-acquired versus healthcare-associated strains. J Antimicrob Chemother. 2004;53:474-9. 
26. Aygen B, Yoruk A, Yyldyz O, Alp E, Kocagoz S, Sumerkan B, et al. Bloodstream infections caused by Staphylococcus aureus in a university hospital in Turkey: clinical and molecular epidemiology of methicillin-resistant Staphylococcus aureus. Clin Microbiol Infect. 2004;10:309-14.

27. Lesens O, Hansmann Y, Brannigan E, Hopkins S, Meyer P, O'Connel B, et al. Healthcare-associated Staphylococcus aureus bacteremia and the risk for methicillin resistance: is the Centers for Disease control and Prevention definition for community acquired bacteremia still appropriate? Infect Control Hosp Epidemiol. 2005;26:204-9.

28. Engemann JJ, Carmeli Y, Cosgrove SE, Fowler VG, Bronstein MZ, Trivette SL, et al. Adverse clinical and economic outcomes attributable to methicillin resistance among patients with Staphylococcus aureus surgical site infection. Clin Infect Dis. 2003;36:592-8.

29. McHugh CG, Riley LW. Risk factors and costs associated with methicillin-resistant Staphylococcus aureus bloodstream infections. Infect Control Hosp Epidemiol. 2004;25:425-30.

30. Lodise T, McKinnon P. Clinical and economic impact of methicillin resistance in patients with Staphylococcus aureus bacteremia. Diagn Microbiol Infect Dis. 2005;52:113-22.

31. Cosgrove SE, Qi Y, Kaye KS, Harbarth S, Karchmer AW, Carmeli Y. The impact of methicillin resistance in Staphylococcus aureus bacteremia on patient outcomes: mortality, length of stay and hospital charges. Infect Control Hosp Epidemiol. 2005;26:166-74.

32. Lowy FD. Antimicrobial resistance: the example of Staphylococcus aureus. J Clin Invest. 2003;111: 1265-73

33. Moreillon P, Que Y, Glauser M. Staphylococcus aureus (Including Staphylococcal Toxic Shock). En: Mandell G, Bennett J, Dolin R, editores. Principles and Practice of Infectious Diseases. Sexta edición; New York: Churchill Livingston; 2005. p.2321-51.

34. Deresinski S. Methicillin-resistant Staphylococcus aureus: An evolutionary, epidemiologic and therapeutic odyssey. Clin Infect Dis. 2005;40:562-73.

35. OIsen JE, Christensen H, Aarestrup FM. Diversity and evolution of blaZ from Staphylococcus aureus and coagulase-negative staphylococci. J Antimicrob Chemother. 2006;57:450-60.

36. Zhang HZ, Hackbarth CJ, Chansky KM, Chambers HF. A proteolytic transmembrane signaling pathway and resistance to beta-lactams in staphylococci. Science. 2001;291:1962-5.

37. Kluytmans-VandenBergh MF, Kluytmans JA. Community-acquired methicillin-resistant Staphylococcus aureus: current perspectives. Clin Microbiol Infect. 2006;12(Suppl.1):9-15.
38. Gomes AR, Sanches IS, Aires de Sousa M, Castañeda E, de Lencastre H. Molecular epidemiology of methicillin-resistant Staphylococcus aureus in Colombian hospitals: dominance of a single unique multidrug resistant clone. Microb Drug Resist. 2001;7:23-32.

39. Aires de Sousa M, Miragaia M, Sanches IS, Avila S, Adamson I, Casagrande ST, et al. Three-year assesment of methicillin-resistant Staphylococcus aureus clones in Latin America from 1996 to 1998. J Clin Microbiol. 2001;39:2197-205.

40. Cruz C, Moreno J, Renzoni A, Hidalgo M, Reyes J, Schrenzel J, et al. Tracking methicillin-resistant Staphylococcus aureus clones in Colombian hospitals over 7 years (1996-2003): emergence of a new dominant clone. Int J Antimicrob Agents. 2005;26:457-62.

41. Sanders CC. Mechanisms responsible for cross resistance and dichotomous resistance among quinolones. Clin Infect Dis. 2001;32(Suppl.1):S1-8.

42. Smith TI, Pearson ML, Wilcox KR, Cruz C, Lancaster MV, Robinson-Dunn B, et al. Emergence of vancomycin resistance in Staphylococcus aureus. $\mathrm{N}$ Eng $\mathrm{J}$ Med. 1999;340:492-501.

43. Tenover FC, Weigel LM, Appelbaum PC, McDougal LK, Chaitram J, McAllister S, et al. Vancomycin-resistant Staphylococcus aureus isolate from a patient in Pennsylvania. Antimicrob Agents Chemother. 2004;48:275-80.

44. Contreras G, Gómez C, Leal A, González MP, Navarrete M. Staphylococcus aureus resistente a la vancomicina: una nueva amenaza. Infectio. 2005;9:91-9.

45. Appelbaum PC, Bozdogan B. Vancomycin resistance in Staphylococcus aureus. Clin Lab Med. 2004;24:381402.

46. Srinivasan A, Dick JD, Perl TM. Vancomycin resistance in staphylococci. Clin Microbiol Rev. 2000; 15:430-8.

47. Smith TL, Pearson M, Wilcox KR, Cruz C, Lancaster M, Robinson-Dunn B, et al. Emergence of vancomycin resistance in Staphylococcus aureus. Glycopeptide-intermediate Staphylococcus aureus Working Group. N Eng J Med. 1999;340:493-501.

48. Garner JS, Jarvis WR, Emori TG, Horan TC, Hughes JM. CDC definitions for nosocomial infections, 1988. Am J Infect Control. 1988;16:128-40.

49. Blyth CC, Darragh H, Whelan A, O'Shea JP, Beaman MH, McCarthy JS. Evaluation of clinical guidelines for managenement of Staphylococcus aureus bactereaemia. Intern Med J. 2002;32:224-32.

50. Mermel LA, Farr BM, Sherertz RJ, Raad II, O'Grady $\mathbf{N}$, Harris JS, et al. Guidelines for the management of intravascular catheter-related infections. Clin Infect Dis. 2001;32:1249-72. 
51. Gopal AK, Fowler VG Jr, Shah M, Gesty-Palmer D, Marr KA, MacClelland RS, et al. Prospective analysis of Staphylococcus aureus in nonneutropenic adults with malignancy. J Clin Oncol. 2000;18:1110-5.

52. Fowler VG Jr, Justice A, Moore C, Benjamin DK Jr, Woods CW, Campbell S, et al. Risk factors for hematogenous complications of intravascular catheterasociated Staphylococcus aureus bacteremia. Clin Infect Dis. 2005;40:695-703.

53. Melzer M, Eykyn SJ, Gransden WR, Chinn S. Is methicillin-resistant Staphylococcus aureus more virulent than methicillin -susceptible $S$. aureus? A comparative cohort study of British patients with nosocomial infection and bacteremia. Clin Infect Dis. 2003;37:1453-60.

54. Pan A, Carnevale G, Catenazzi P, Colombini P, Crema L, Dolcetti L, et al. Trends in methicillin-resistant Staphylococcus aureus (MRSA) bloodstream infections: effect of the MRSA "search and isolate" strategy in a hospital in Italy with hyperendemic MRSA. Infect Control Hosp Epidemiol. 2005;26:127-33.

55. Talon D, Woronoff-Lemsi M, Limat S, Bertrand $\mathbf{X}$, Chatillon M, Gil H, et al. The impact of resistance to methicillin in Staphylococcus aureus bacteremia on mortality. Eur J Intern Med. 2002;13:31-6.

56. Lowy FD. Staphylococcus aureus infections. N Eng J Med. 1998;339:520-32.

57. McClelland RS, Fowler VG Jr, Sanders LL, Gottlieb G, Kong LK, Sexton DJ, et al. Staphylococcus aureus bacteremia among elderly vs younger adult patients. Comparison of clinical features and morality. Arch Intern Med. 1999;159:1244-7.

58. Lesens $\mathbf{O}$, Methlin C, Hansmann Y, Remy V, Martinot M, Bergin C, et al. Role de comorbidity in mortality related to Staphylococcus aureus bacteriemia: a prospective study using the Charlson weighted index of comorbidity. Infect Control Hosp Epidemiol. 2003;24:890-6

59. Ladhani S, Konana OS, Mwarumba S, English MC. Bacteraemia due to Staphylococcus aureus. Arch Dis Child. 2004;89:568-71.

60. Johnson LB, Almoujahed M, Ilg K, Maolood L, Khatib R. Staphylococcus aureus bacteremia: compliance with standard treatment, long-term outcome and predictors of relapse. Scand J Infect Dis. 2003;35:782-9.

61. Kim SH, Park WB, Lee KD, Kang Cl, Bang JW, Kim $\mathrm{HB}$, et al. Outcome of inappropriate initial antimicrobial treatment in patients with methicillin resistant Staphylococcus aureus bacteraemia. J Antimicrob Chemother. 2004;54:489-97.

62. Chang FY, Peacock JE, Musher DM, Triplett P, MacDonald BB, Mylotte JM, et al. Staphylococcus aureus bacteremia. Recurrence and the impact of antibiotic treatment in a prospective multicenter study. Medicine (Baltimore). 2003;82:333-9.
63. Lodise TP, Mckinnon PS, Swiderski L, Rybak MJ. Outcomes analysis of delayed antibiotic treatment for hospital-adquired Staphylococcus aureus bacteremia. Clin Infect Dis. 2003;36:1418-23.

64. Cosgrove S, Sakoulas G, Perencevich E, Schwaber M, Karchmer A, Carmeli Y. Comparison of mortality associated with methicillin-resistant and methicillin-susceptible Staphylococcus aureus bacteremia: a metaanalysis. Clin Infec Dis. 2003;36:53-9.

65. Rodriguez-Bano J. Selection of empiric therapy in patients with catheter-related infections. Clin Microbiol Infect. 2002;8:275-81.

66. Fatkenheuer G, Cornely O, Seifert H. Clinical management of catheter-related infections. Clin Microbiol Infect. 2002;8:545-50.

67. Charlebois ED, Perdreau-Remington F, Kreiswirth B, Bangsberg DR, Ciccarone D, Diep BA, et al. Origins of community strains of methicillin-resistant Staphylococcus aureus. Clin Infect Dis. 2004;39:47-54.

68. Ellis MW, Lewis JS 2nd. Treatment approaches for community-acquired methicillin resistant Staphylococcus aureus infections. Curr Opin Infect Dis. 2005;18:496-501.

69. Schentag JJ. Antimicrobial management strategies for Gram-positive bacterial resistance in the intensive care unit. Crit Care Med. 2001;29 (Suppl. 4):100-7.

70. Jensen A. Treatment of Staphylococcus aureus bacteremia and secondary infections. Antimicrob Infect Dis News. 2000;18:73-5.

71. Hsu RB, Chu SH. Impact of methicillin resistance on clinical features and outcomes of infective endocarditis due to Staphylococcus aureus. Am J Med Sci. 2004;328:150-5

72. Li JS, Sexton DJ, Mick N, Nettles R, Fowler VG Jr, Ryan $\mathbf{T}$, et al. Proposed modifications to the Duke criteria for the diagnosis of infective endocarditis. Clin Infect Dis. 2000;30:633-8.

73. Anstead GM, Owens AD. Recent advances in the treatment of infections due to resistant Staphylococ cus aureus. Curr Opin Infect Dis. 2004;17:549-55.

74. Lesens O, Hansmann Y, Storck D, Christmann D. Risk factors for metastatic infection in patients with Staphylococcus aureus bacteremia with and without endocarditis. Eur J Intern Med. 2003;14:227-31.

75. Stevens DL, Herr D, Lampiris H, Hunt JL, Betts DH, Hafkin B, et al. Linezolid versus vancomycin for the treatment of methicillin-resistant Staphylococcus aureus infections. Clin Infect Dis. 2002;34:1481-90.

76. Cabell CH, Fowler VG Jr. Importance of aggressive evaluation in patients with Staphylococcus aureus bacteremia. Am Heart J. 2004;147:379-80.

77. DeRyke CA, Lodise TP Jr, Rybak MJ, McKinnon PS. Epidemiology, treatment, and outcomes of 
nosocomial bacteremic Staphylococcus aureus pneumonia. Chest. 2005;128:1414-22.

78. Daum R, Seal J. Evolving antimicrobial chemotherapy for Staphylococcus aureus infections: our backs to the wall. Crit Care Med. 2001;29(Suppl.4):92-6.

79. Lundstrom TS, Sobel JD. Antibiotics for gram-positive bacterial infections: vancomycin, quinupristindalfopristin, linezolid, and daptomycin. Infect Dis Clin North Am. 2004;18:651-68.

80. Marshall C, Kossmann T, Wesselingh S, Spelman D. Methicillin-resitant Staphylococcus aureus and beyond: what's new in the world of the golden staph? ANZ J Surg. 2004;74:465-9.

81. Stevens DL, Herr D, Lampiris H, Hunt JL, Batts DH, Hafkin B. Linezolid versus vancomycin for the treatment of methicillin-resistant Staphylococcus aureus infections. Clin Infect Dis. 2002;34:1481-90.

82. Howden BP, Ward PB, Charles PG, Korman TM, Fuller A, du Cros P, et al. Treatment outcomes for serious infections caused by methicillin-resistant Staphylococcus aureus with reduced vancomycin susceptibility. Clin Infec Dis. 2004;38:521-8.

83. Kalil AC, Puumala SE, Stoner J. Unresolved questions with the use of linezolid vs vancomycin for nosocomial pneumonia. Chest. 2004;125:2370-1.

84. Jensen AG, Espersen F, Skinhoj P, Frimodt-Moller N. Bacteremic Staphylococcus aureus spondilytis. Arch Intern Med. 1998;158:509-17.

85. Belkum AV, Verbrugh H. 40 years of methicillin resistant Staphylococcus aureus. $\mathrm{Br}$ Med $\mathrm{J}$. 2001;323:644-5. 